\title{
Design of Stable IIR Digital Filters With Equiripple Passbands and Peak-Constrained Least-Squares Stopbands
}

\author{
W.-S. Lu, Fellow, IEEE
}

\begin{abstract}
This paper describes an algorithmic development for the design of stable infinite impulse response (IIR) digital filters with equiripple passbands and peak-constrained leastsquares stopbands. Central to the development is a re-formulation of the design problem as an iterative quadratic programming problem where the stability and equiripple passbands and peakconstrained requirements are met by imposing a system of linear inequality constraints while the LSS property is satisfied by minimizing a weighted least-squares type objective function.
\end{abstract}

\section{INTRODUCTION}

$\mathbf{R}$ ECURSIVE digital filters offer improved selectivity, computational efficiency, and reduced system delay compared to what can be achieved by nonrecursive digital filters of comparable approximation accuracy [1]-[11]. A class of finite impulse response (FIR) filters with equiripple passband and peak-constrained least-square stopband (EPPCLSS) has been investigated in detail by Adams, et al. [12]-[15]. This brief describes an algorithmic development for the design of stable infinite impulse response (IIR) digital filters with EPPCLSS, using an iterative quadratic programming $(\mathrm{QP})$ approach initiated in [16]. In the design, the stability and equiripple passband and peak-constrained (EPPC) requirements are met by imposing a system of linear inequality constraints while the LSS property is satisfied by employing a weighted leastsquares type objective function. An early version of this work was reported in [17].

\section{PROBLEM Formulation}

\section{A. Problem Statement}

Let $h_{d}(\omega)$ be the desired frequency response of bandpass nature, and for simplicity, we assume that $h_{d}(\omega)$ has one passband and one stopband. We seek to find a causal IIR transfer function of order $n$, i.e.,

$$
h(z)=\frac{b(z)}{\hat{d}(z)}=\frac{\sum_{i=0}^{n} b_{i} z^{n-i}}{z^{n-r} \sum_{i=0}^{r} d_{i} z^{r-i}}, \quad \text { with } d_{0}=1
$$

Manuscript received September 4, 1998; revised August 1999. This work was supported by the National Sciences and Engineering Council of Canada. This paper was recommended by Associate Editor P. Diniz.

The author is with the Department of Electrical and Computer Engineering, University of Victoria, Victoria, BC, V8W 3P6 Canada.

Publisher Item Identifier S 1057-7130(99)09231-9. such that the weighted error

$$
J(\mathbf{x})=\frac{1}{2} \int_{-\pi}^{\pi} W(\omega)\left|h_{d}(\omega)-h(\omega)\right|^{2} d \omega
$$

is minimized subject to the following constraints.

1) $h(z)$ is stable, i.e.,

$$
\sum_{i=0}^{r} d_{i} z^{r-i} \neq 0, \quad \text { for }|z| \geq 1 .
$$

2) $h(z)$ is peak-constrained in the stopband $\mathcal{S}_{a}$, i.e.,

$$
|h(\omega)| \leq \delta_{a}, \quad \text { for } \omega \in \mathcal{S}_{a} .
$$

3) $|h(z)|$ is equiripple over the passband $\mathcal{S}_{p}$. It is known that [12] with properly chosen weight $W(\omega), h(\omega)$ becomes equiripple in the passband $\mathcal{S}_{p}$ if

$$
|| h(\omega)|-1| \leq \delta_{p}, \quad \omega \in \mathcal{S}_{p} .
$$

Note that the denominator polynomial $\hat{d}(z)$ in (1) has the form

$$
\hat{d}(z)=z^{n-r} d(z), \quad 0 \leq r \leq n
$$

with

$$
d(z)=\sum_{i=0}^{r} d_{i} z^{r-i}, \quad d_{0}=1 .
$$

This form of $\hat{d}(z)$ is convenient to reflect the fact that assigning a certain number of zeros at the origin may be beneficial for the design of several types of digital filters [18]. Note that the number of parameters contained in $\hat{d}(z)$ is $r$ which can vary from zero to $n$, depending on how many poles of $h(z)$ one wishes to assign to the origin.

\section{B. Formulating the Design as an Iterative QP Problem}

The vector $\mathrm{x}$ in (2) collects the coefficient vectors of $d(z)$ and $b(z)$, denoted by $\mathbf{d}$ and $\mathbf{b}$ respectively, as $\mathbf{x}=\left[\begin{array}{ll}\mathbf{d}^{T} \mathbf{b}^{T}\end{array}\right]^{T}$. We write $h(\omega)=b(\omega) / d(\omega)$ with

$$
b(\omega)=\sum_{i=0}^{n} b_{i} e^{-i j \omega}, \quad d(\omega)=\sum_{i=0}^{r} d_{i} e^{-i j \omega}
$$

and $J(\mathbf{x})$ in (2) as

$$
J(\mathbf{x})=\frac{1}{2} \int_{-\pi}^{\pi} \hat{W}(\omega)\left|h_{d}(\omega) d(\omega)-b(\omega)\right|^{2} d \omega
$$


where $\hat{W}(\omega)=W(\omega) /|d(\omega)|^{2}$. Based on (7), the $k$ th iteration of an iterative-QP-based EPPCLSS design minimizes the quadratic error function

$$
J^{(k)}\left(\mathbf{x}_{k}\right)=\frac{1}{2} \int_{-\pi}^{\pi} \hat{W}_{k-1}(\omega)\left|h_{d}(\omega) d^{(k)}(\omega)-b^{(k)}(\omega)\right|^{2} d \omega
$$

subject to the constraints in (3)-(5) with $h(\omega)$ replaced by $h^{(k)}(\omega)$, where $\hat{W}_{k-1}(\omega)=W(\omega) /\left|d^{(k-1)}(\omega)\right|^{2}$. It should be pointed out that the weighting modification procedure given in (7) and (8) is closely connected to the Steiglitz-McBride approach which finds applications in system modeling, identification, and adaptive filtering [19].

Let $\mathbf{d}_{k}=\left[\begin{array}{llll}d_{1}^{(k)} & \cdots & d_{r}^{(k)}\end{array}\right]^{T}$ and $\mathbf{b}_{k}=\left[\begin{array}{lll}b_{0}^{(k)} & \cdots & b_{n}^{(k)}\end{array}\right]^{T}$ be the coefficient vectors of $d^{(k)}(\omega)$ and $b^{(k)}(\omega)$, respectively, and let $\Omega_{1}=\left[\begin{array}{lll}e^{-j \omega} & \cdots & e^{-j r \omega}\end{array}\right]^{T}$ and $\Omega_{0}=$ $\left[\begin{array}{llll}1 & e^{-j \omega} & \cdots & e^{-j n \omega}\end{array}\right]^{T}$. Then $d^{(k)}(\omega)=1+\boldsymbol{\Omega}_{1}^{T} \mathbf{d}_{k}$, $b^{(k)}(\omega)=\Omega_{0}^{T} \mathbf{b}_{k}$, and $J^{(k)}\left(\mathrm{x}_{k}\right)$ in (8) can be expressed as

$$
J^{(k)}\left(\mathbf{x}_{k}\right)=\frac{1}{2} \mathbf{x}_{k}^{T} \mathbf{H}_{k} \mathbf{x}_{k}+\mathbf{x}_{k}^{T} \mathbf{p}_{k}+\text { constant }
$$

where

$$
\mathbf{x}_{k}=\left[\begin{array}{l}
\mathbf{d}_{k} \\
\mathbf{b}_{k}
\end{array}\right], \quad \mathbf{H}_{k}=\left[\begin{array}{ll}
\mathbf{H}_{11 k} & \mathbf{H}_{12 k} \\
\mathbf{H}_{12 k}^{T} & \mathbf{H}_{22 k}
\end{array}\right], \quad \text { and } \quad \mathbf{p}_{k}=\left[\begin{array}{l}
\mathbf{p}_{1 k} \\
\mathbf{p}_{2 k}
\end{array}\right]
$$

with

$$
\begin{aligned}
\mathbf{H}_{11 k} & =\int_{-\pi}^{\pi} \hat{W}_{k-1}(\omega)\left|h_{d}(\omega)\right|^{2}\left(\boldsymbol{\Omega}_{1} \boldsymbol{\Omega}_{1}^{H}\right) d \omega \\
\mathbf{H}_{12 k} & =-\int_{-\pi}^{\pi} \hat{W}_{k-1}(\omega) \operatorname{Re}\left[h_{d}(\omega) \boldsymbol{\Omega}_{1} \boldsymbol{\Omega}_{0}^{H}\right] d \omega \\
\mathbf{H}_{22 k} & =\int_{-\pi}^{\pi} \hat{W}_{k-1}(\omega)\left(\boldsymbol{\Omega}_{0} \boldsymbol{\Omega}_{0}^{H}\right) d \omega \\
\mathbf{p}_{1 k} & =\int_{-\pi}^{\pi} \hat{W}_{k-1}(\omega)\left|h_{d}(\omega)\right|^{2} \operatorname{Re}\left(\boldsymbol{\Omega}_{1}\right) d \omega \\
\mathbf{p}_{2 k} & =-\int_{-\pi}^{\pi} \hat{W}_{k-1}(\omega) \operatorname{Re}\left[h_{d}(\omega) \overline{\boldsymbol{\Omega}}_{0}\right] d \omega .
\end{aligned}
$$

\section{DESIGN CONSTRAINTS}

For the sake of notation simplicity, in the rest of the paper only low-pass filters with approximately constant group delay in the passband are considered. The normalized passband and stopband edges are denoted by $\omega_{p}$ and $\omega_{a}$, respectively.

\section{A. Stability}

The stability of $d^{(k)}(z)$ is guaranteed if

$$
\operatorname{Re}\left[d^{(k)}(\omega)\right] \geq \delta>0, \quad \text { for } \omega \in[-\pi, \pi]
$$

where $\delta>0$ is a lower bound to ensure a reasonable stability margin [20]. In practice, a discrete version of (10) is implemented, i.e., $\operatorname{Re}\left[d^{(k)}(\omega)\right] \geq \delta>0$, for $\omega \in \mathcal{S}_{s}=$ $\left\{\omega_{i}^{(s)}, i=1, \ldots, n_{s}\right\}$. This leads to a set of linear constraints on $\mathrm{x}_{k}$

$$
\mathbf{A}_{s} \mathbf{x}_{k} \leq(1-\delta) \mathbf{e}_{n_{s}}
$$

where

$$
\begin{aligned}
\mathbf{A}_{s} & =-\left[\begin{array}{cccc}
\cos \omega_{1}^{(s)} & \cdots & \cos n \Omega_{1}^{(s)} & \\
\vdots & & \vdots & 0 \\
\cos \omega_{n_{s}}^{(s)} & \ldots & \cos n \Omega_{n_{s}}^{(s)}
\end{array}\right]_{n_{s} \times(n+r+1)} \\
\mathbf{e}_{n_{s}} & =\left[\begin{array}{llll}
1 & 1 & \ldots & 1
\end{array}\right]_{n_{s} \times 1}^{T}
\end{aligned}
$$

It should be stressed that the constraint in (10) is a sufficient condition to assure the stability. A nice feature of (10) is that it is a linear constraint. However, design simulations have indicated that stable candidate transfer functions with excellent performance may be excluded from the design procedure simply because condition (10) is often too restrictive. The discrete version of the condition, namely (11), offers some flexibility in controlling filter's stability. On one hand, with a dense $\mathcal{S}_{s}$, the constraint in (11) can be made arbitrarily close to (10) so as to guarantee the stability. On the other hand, using (11) with a relatively sparse $\mathcal{S}_{s}$ turns out to be an effective and less restrictive way to control the filter's stability. As a matter of fact, simulations have indicated that for a polynomial $d(z)$ of order $r$, the constraint in (11) with an $n_{s} \leq 2 r$ is usually sufficient to yield a stable design. We shall come back to this point in Section $\mathrm{V}$ where two design examples are discussed in detail.

In a recent paper by Lang [18], Rouché's theorem on the number of zeros of two analytic functions was employed to deduce a stability constraint, which appears to be less conservative than (10)

\section{B. Weighting Function $W(\omega)$}

In the sequel, we shall use a piecewise-constant weighting function

$$
W(\omega)= \begin{cases}1, & \text { for }|\omega| \leq \omega_{p} \\ w, & \text { for } \omega_{a} \leq|\omega| \leq \pi\end{cases}
$$

With a sufficiently large $w$ and a set of amplitude constraints on $\left[-\omega_{p}, \omega_{p}\right]$, the filter obtained becomes nearly equiripple in the passband region [12].

\section{Amplitude Constraints in Passband}

Suppose the algorithm converges. Then for an $\omega \in\left[0, \omega_{p}\right]$ and sufficiently large $k$, we have $h(\omega) \approx b^{(k)}(\omega) / d^{(k-1)}(\omega) \approx$ $e^{-j K \omega} R(\omega)$, where $K$ denotes the desired group delay of the filter in the passband, and $R(\omega)=\left|b^{(k)}(\omega)\right| /\left|d^{(k-1)}(\omega)\right| \approx$ $|h(\omega)|$. If we write $d^{(k-1)}(\omega)=e^{j \theta_{k-1}(\omega)}\left|d^{(k-1)}(\omega)\right|$ where $\theta_{k-1}(\omega)$ is the phase response of $d^{(k-1)}(\omega)$, then we have

$$
\begin{aligned}
R(\omega) & \approx e^{j K \omega} \frac{b^{(k)}(\omega)}{d^{(k-1)}(\omega)} \\
& =e^{j\left(K \omega-\theta_{k-1}(\omega)\right)} \frac{b^{(k)}(\omega)}{\left|d^{(k-1)}(\omega)\right|} \\
& =\frac{\mathbf{c}_{k}^{T}(\omega) \mathbf{b}_{k}}{\left|d^{(k-1)}(\omega)\right|}
\end{aligned}
$$

where the last equality is because $R(\omega)$ is real, and

$$
\begin{array}{r}
\mathbf{c}_{k}(\omega)=\left[\cos \left(K \omega-\theta_{k-1}(\omega)\right)\right. \\
\left.\cdots \cos \left((K-n) \omega-\theta_{k-1}(\omega)\right)\right]^{T} .
\end{array}
$$


By (5) and the definition of $R(\omega)$, we see that (5) approximately holds if $|R(\omega)-1| \leq \delta_{p}$. This, in conjunction with (13), leads to a discrete version of the amplitude constraints on $\left[0, \omega_{p}\right]$ as

$$
\mathbf{A}_{p}^{(k)} \mathbf{X}_{k} \leq \mathbf{q}_{p}^{(k)}
$$

where

$$
\begin{aligned}
\mathbf{A}_{p}^{(k)}= & {\left[\begin{array}{c}
\mathbf{c}_{k}^{T}\left(\omega_{1}^{(p)}\right) \\
\vdots \\
\mathbf{c}_{k}^{T}\left(\omega_{n_{p}}^{(p)}\right) \\
\mathbf{0}-\mathbf{c}_{k}^{T}\left(\omega_{1}^{(p)}\right) \\
\vdots \\
-\mathbf{c}_{k}^{T}\left(\omega_{n_{p}}^{(p)}\right)
\end{array}\right] } \\
\mathbf{q}_{p}^{(k)}= & {\left[\begin{array}{c}
\left(1+\delta_{p}\right)\left|d^{(k-1)}\left(\omega_{1}^{(p)}\right)\right| \\
\vdots \\
\left(1+\delta_{p}\right)\left|d^{(k-1)}\left(\omega_{n_{p}}^{(p)}\right)\right| \\
\left(\delta_{p}-1\right)\left|d^{(k-1)}\left(\omega_{1}^{(p)}\right)\right| \\
\vdots \\
\left(\delta_{p}-1\right)\left|d^{(k-1)}\left(\omega_{n_{p}}^{(p)}\right)\right|
\end{array}\right] }
\end{aligned}
$$

with $\mathcal{S}_{p}=\left\{\omega_{i}^{(p)}, i=1, \ldots, n_{p}\right\}$ a set of grid points in the passband.

\section{Amplitude Constraints in the Stopband}

The peak constraints in the stopband can be expressed approximately as $\left|b^{(k)}(\omega)\right| /\left|d^{(k-1)}(\omega)\right| \leq \delta_{a}$ for $\omega \epsilon$ $\mathcal{S}_{a}$. Let $\mathbf{c}(\omega)=\left[\begin{array}{llll}1 & \cos \omega & \cdots & \cos n \omega\end{array}\right]^{T}$ and $\mathbf{s}(\omega)=$ $\left[\begin{array}{llll}0 & \sin \omega & \cdots & \sin n \omega\end{array}\right]^{T}$, it can easily be verified that the above constraints are satisfied if

$$
\begin{aligned}
\left|\mathbf{c}^{T}(\omega) \mathbf{b}_{k}\right| & \leq \frac{1}{2} \delta_{a}\left|d^{(k-1)}(\omega)\right| \\
\left|\mathbf{s}^{T}(\omega) \mathbf{b}_{k}\right| & \leq \frac{1}{2} \delta_{a}\left|d^{(k-1)}(\omega)\right| .
\end{aligned}
$$

Imposing the constraints in (15) on the set of grid points $\mathcal{S}_{a}=\left\{\omega_{i}^{(a)}, i=1, \ldots, n_{a}\right\}$ in the stopband, we obtain

$$
\mathbf{A}_{a}^{(k)} \mathbf{X}_{k} \leq \mathbf{q}_{a}^{(k)}
$$

where

$$
\mathbf{A}_{a}^{(k)}=\left[\begin{array}{r}
\mathbf{C}(\omega) \\
-\mathbf{C}(\omega) \\
\mathbf{S}(\omega) \\
-\mathbf{S}(\omega)
\end{array}\right], \quad \mathbf{q}_{a}^{(k)}=\frac{\delta_{a}}{2}\left[\begin{array}{l}
\mathbf{D}(\omega) \\
\mathbf{D}(\omega) \\
\mathbf{D}(\omega) \\
\mathbf{D}(\omega)
\end{array}\right]
$$

with

$$
\begin{aligned}
\mathbf{C}(\omega) & =\left[\begin{array}{c}
\mathbf{c}^{T}\left(\omega_{1}^{(a)}\right) \\
\vdots \\
\mathbf{c}^{T}\left(\omega_{n_{a}}^{(a)}\right)
\end{array}\right], \quad \mathbf{S}(\omega)=\left[\begin{array}{c}
\mathbf{s}^{T}\left(\omega_{1}^{(a)}\right) \\
\vdots \\
\mathbf{s}^{T}\left(\omega_{n_{a}}^{(a)}\right)
\end{array}\right] \\
\mathbf{D}(\omega) & =\left[\begin{array}{c}
\left|d^{(k-1)}\left(\omega_{1}^{(a)}\right)\right| \\
\vdots \\
\left|d^{(k-1)}\left(\omega_{n_{a}}^{(a)}\right)\right|
\end{array}\right] .
\end{aligned}
$$

TABLE I

Zeros and Poles of the Transfer Function in Example 1

\begin{tabular}{c|c|c}
\hline$b_{0}$ & zeros & poles \\
\hline-0.00046047527 & 18.63131093 & 0.20628660 \\
& $1.49675301 \pm 0.48221524 j$ & $0.055677115 \pm 0.55763187 j$ \\
& $0.89762684 \pm 1.22910309 j$ & $-0.032471477 \pm 0.93555574 j$ \\
& $-0.99895376 \pm 0.20015069 j$ & plus another 10 \\
& $-0.20222995 \pm 0.97998167 j$ & poles at the origin \\
& $-0.84535803 \pm 0.56243645 j$ & \\
& $-0.35458606 \pm 0.93926134 j$ & \\
\hline & $-0.59858640 \pm 0.81318261 j$ & \\
\hline
\end{tabular}

\section{A Design Algorithm}

In summary, we propose an iterative QP problem where the objective function in the $k$ th iteration is given by

$$
J^{(k)}\left(\mathbf{x}_{k}\right)=\frac{1}{2} \mathbf{x}_{k}^{T} \mathbf{H}_{k} \mathbf{x}_{k}+\mathbf{x}_{k}^{T} \mathbf{p}_{k}+\text { constant }
$$

with $\mathrm{x}_{k}, \mathbf{H}_{k}$, and $\mathbf{p}_{k}$, specified in (9). The stability and EPPCLSS properties of the filter are assured by imposing the linear constraints

$$
\mathbf{A}_{k} \mathbf{x}_{k} \leq \mathbf{q}_{k}
$$

and using a sufficiently large weight $w$, where $\mathbf{A}_{k}$ in (18) combines $\mathbf{A}_{s}$ in (11), $\mathbf{A}_{p}^{(k)}$ in (14) and $\mathbf{A}_{a}^{(k)}$ in (16) as

$$
\begin{aligned}
\mathbf{A}_{k} & =\left[\begin{array}{c}
\mathbf{A}_{s} \\
\mathbf{A}_{p}^{(k)} \\
\mathbf{A}_{a}^{(k)}
\end{array}\right] \text { and } \mathbf{q}_{k} \text { in (18) is given by } \\
\mathbf{q}_{k} & =\left[\begin{array}{c}
(1-\delta) \mathbf{e}_{n_{s}} \\
\mathbf{q}_{p}^{(k)} \\
\mathbf{q}_{a}^{(k)}
\end{array}\right]
\end{aligned}
$$

The problem of minimizing $J^{(k)}\left(\mathrm{x}_{k}\right)$ in (17) subject to the constraints in (18) is a convex quadratic programming (QP) problem whose solution can be obtained using efficient optimization algorithms [21]. It is worthwhile to note that the optimization toolbox associated with MATLAB includes a command qp that can be used as an efficient QP solver.

Once a solution $\mathrm{x}_{k}$ of the $\mathrm{QP}$ problem

$$
\begin{array}{ll}
\text { minimize } & J^{(k)}\left(\mathbf{x}_{k}\right) \\
\text { subject to } & \mathbf{A}_{k} \mathbf{x}_{k} \leq q_{k}
\end{array}
$$

is obtained, $\mathrm{x}_{k}$ is modified by combining it linearly with $\mathrm{x}_{k-1}$ obtained from the preceding iteration to yield a refined iterate $\mathbf{x}_{k}:=\tau \mathbf{x}_{k}+(1-\tau) \mathbf{x}_{k-1}$ where $0<\tau<1$ is a relaxation parameter. This $\mathrm{x}_{k}$ is then used in the next iteration. The iteration continues until $\left\|\mathrm{x}_{k}-\mathrm{x}_{k-1}\right\|_{2}$ is less than a prescribed tolerance.

A couple of comments on the algorithm's convergence and nature of the solution obtained: The convergence of the Steiglitz-McBride procedure as outlined in (7) and (8) is a fairly involved issue, but there are sufficient evidence indicating that the convergent point(s) of the Steiglitz-McBride procedure can be very close to a global minimum of the least-squares error function in (2) (see [19, ch. 8] for a detailed analysis on this matter). In the present case, the Steiglitz-McBride 


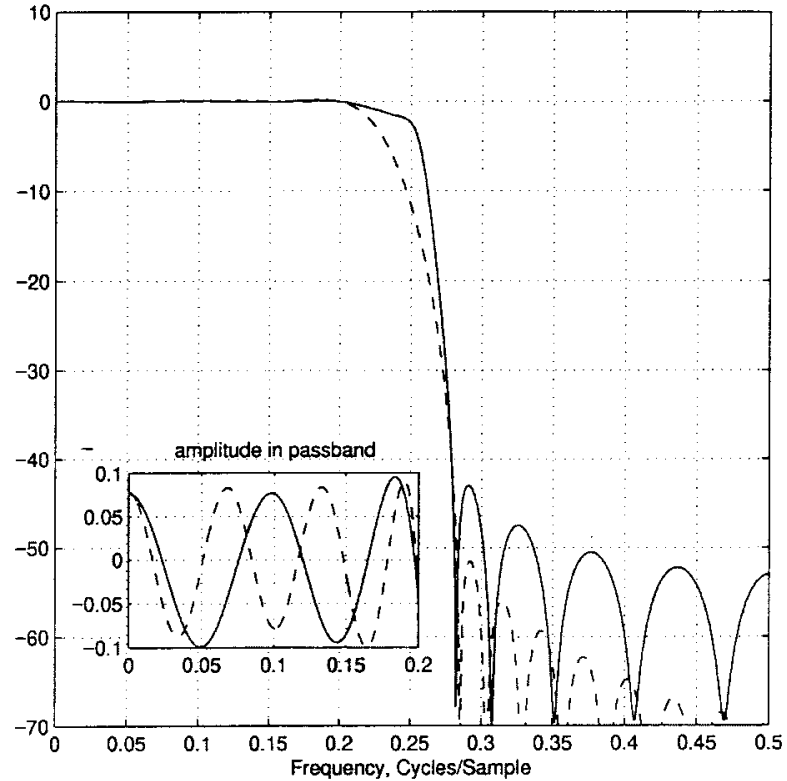

(a)

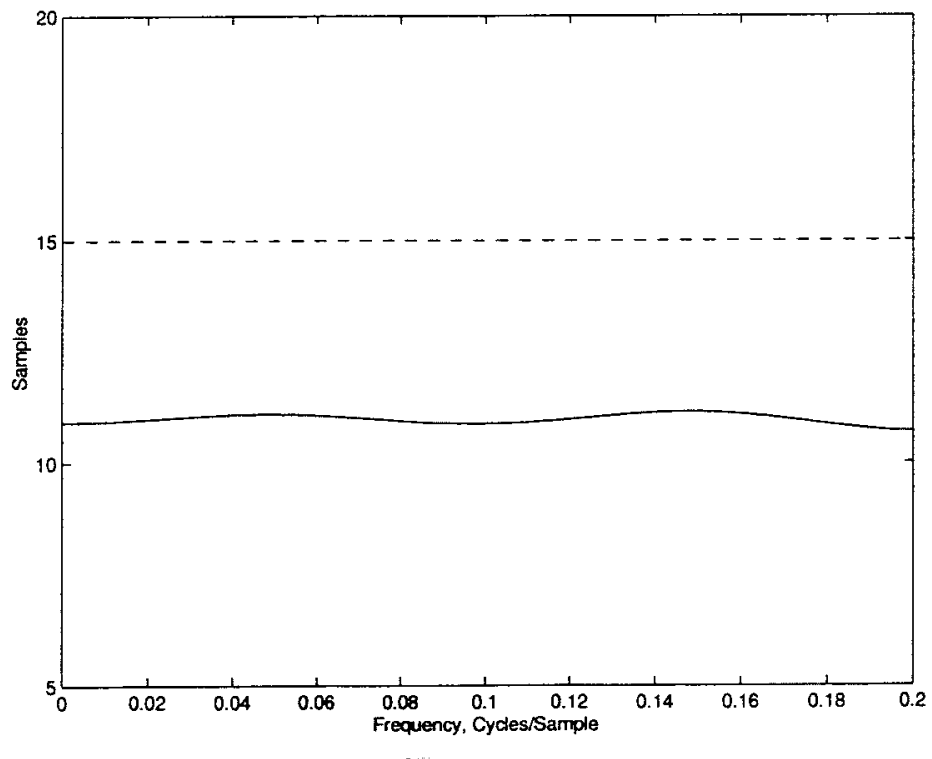

(b)

Fig. 1. Frequency responses of the IIR filter (solid lines) and the FIR filter (dashed lines) in Example 1. (a) Amplitude responses. (b) Group delay in passband.

procedure is reduced to solving a sequence of QP problems in (19). Although a convergence proof of the algorithm is not yet available, our simulation studies have indicated that the algorithm converges whenever the QP problems involved are all solvable. When, for example, the minimum stopband amplitude attenuation and/or maximum passband deviation are too restrictive relative to the filter order, the QP solver would complain of the constraints being overly stringent. In this case, a typical QP solver (e.g., the QP solver in the MATLAB Optimization Toolbox) still produces a "solution," but the proposed algorithm may not converge in such a case. On the other hand, if the QP problems in (19) is solvable for each $k$, then this consistent solvability can be viewed as a strong indication of the existence of a solution with respect to the given design specifications, and in this case it is more than often that the proposed algorithm will converge to a satisfactory design.

It should be stressed that the convex QP problem in (19) is merely a discrete (hence approximate) version of the constrained least-squares optimization problem characterized by (2)-(5). Therefore, a convergent point of the sequence $\left\{\mathbf{x}_{k}\right\}$ obtained by iteratively solving the QP problem in (19) can only be deemed as a suboptimal design.

\section{Design Examples}

We describe below two examples in which the proposed method was used to design stable IIR digital filters. In both designs, the initial point was simply a linear-phase FIR filter of order $n$, where $n=15$ for the first example, and $n=12$ for the second example, designed using a Hamming window. Hence, the initial point can be described as $\mathbf{x}_{0}=\left[\begin{array}{ll}\mathbf{d}_{0}^{T} & \mathbf{b}_{0}^{T}\end{array}\right]^{T}$ with $\mathrm{d}_{0}=\left[\begin{array}{llll}0 & 0 & \ldots & 0\end{array}\right]^{T} \in R^{r}$ and $\mathrm{b}_{0}$ the impulse response of the FIR filter.

Example 1: A stable low-pass IIR filter of order 15 with EPPCLSS was designed to satisfy the following specifications: $\omega_{p}=0.2, \omega_{a}=0.28$, maximum passband amplitude deviation $\leq 0.1 \mathrm{~dB}$, minimum stopband attenuation $\geq 43 \mathrm{~dB}$, group delay in passband $=11$ samples with maximum deviation less than 0.35. With $n=15, r=5, \delta_{p}=0.01, \delta_{a}=0.2$, $w=1000$, and convergence tolerance $\varepsilon=10^{-4}$, it took the proposed algorithm 21 iterations to converge to a solution point. The poles and zeros of the filter obtained are given in Table I. The largest magnitude of the poles is 0.9361 . The amplitude response and group delay (in the passband) are depicted in Fig. 1(a) and (b) (solid lines), respectively. In the design, $\mathbf{H}_{k}$ and $\mathbf{p}_{k}$ in (9) were evaluated using a set of 300 grid points placed evenly in the passband and stopband. The discrete set $\mathcal{S}_{p}$ consists of 30 equally spaced frequency samples in the normalized passband $[0,0.2]$ and set $\mathcal{S}_{a}$ consists of 42 equally spaced frequency samples in the first quarter of the normalized stopband $[0.28,0.5]$. The discrete $\mathcal{S}_{s}$ in this design consists of six equally spaced frequency samples on the entire frequency range $[0,0.5]$ to ensure the filter's stability. From a number of numerical simulations that we conducted to test the proposed design method, it was observed that the stability of the denominator polynomial $d(z)$ can be ensured by imposing $n_{s}$ constrains in (11), where $n_{s}$ need not exceed $2 r$, where $r$ is the order of $d(z)$. Note that these $n_{s}$ constrains do not in general guarantee the sufficient condition in (10) but they are of critical importance in the design process to yield a stable transfer function. The maximum passband amplitude deviation, minimum stopband attenuation, and maximum group-delay deviation in passband are given in Table II.

The design obtained was compared to a linear-phase EPPCLSS FIR filter with 31 taps. The FIR was designed using the method described in [12]. The filter's performance is shown in Fig. 1 (dashed lines) and Table II. It is observed that the performance of these two filters in terms of passband amplitude deviation and stopband amplitude attenuation are 


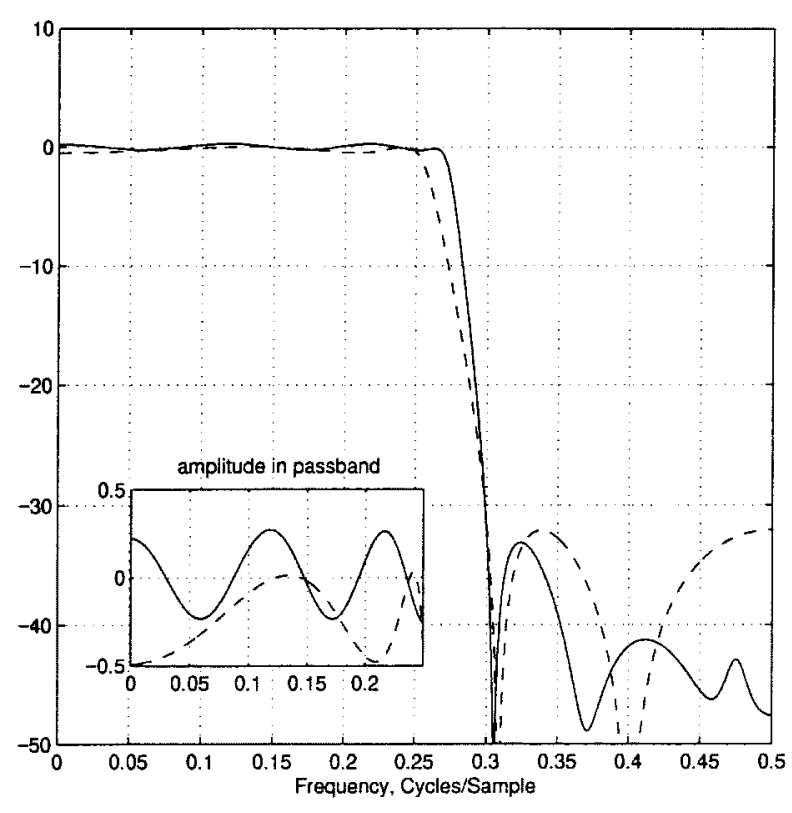

(a)

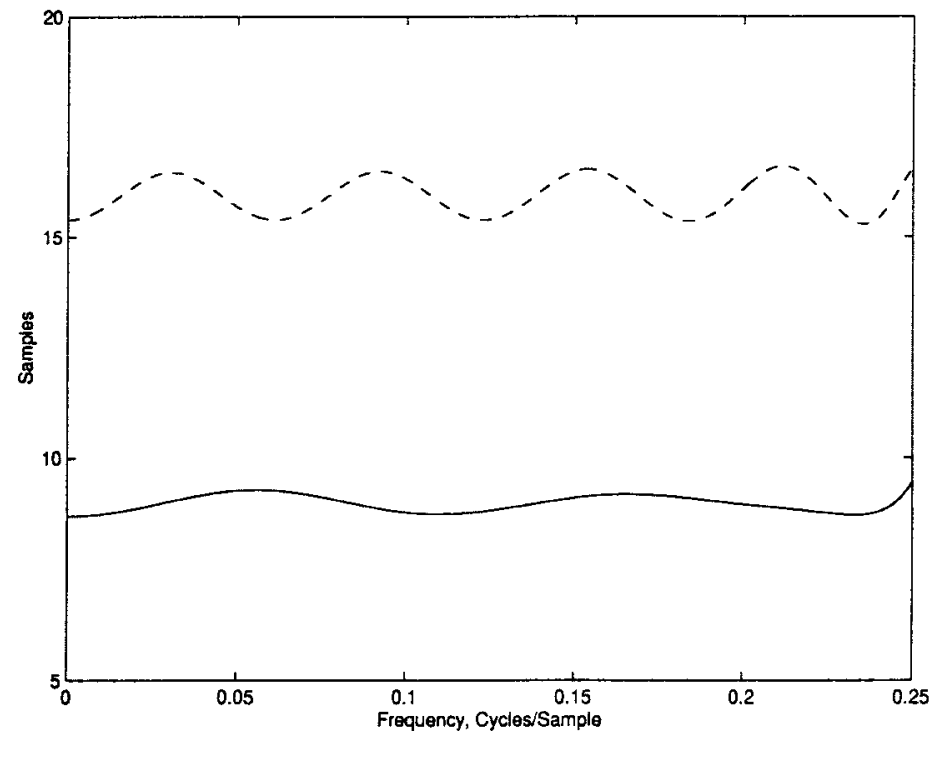

(b)

Fig. 2. Frequency responses of the IIR filter (solid lines) and Deczky's filter (dashed lines) in Example 2. (a) Amplitude responses. (b) Group delay in passband.

TABLE II

PERformance COMPARISONS IN EXAMPLE 1

\begin{tabular}{l|c|c}
\hline Filter & Proposed IIR & Linear Phase FIR \\
\hline $\begin{array}{l}\text { Maximum passband } \\
\text { amplitude deviation (dB) }\end{array}$ & 0.0992 & 0.0995 \\
\hline $\begin{array}{l}\text { Minimum stopband } \\
\text { amplitude attenuation(dB) }\end{array}$ & 43.0046 & 42.0087 \\
\hline $\begin{array}{l}\text { Maximum group delay } \\
\text { deviation in passband } \\
\text { (Samples) }\end{array}$ & 0.3109 & 0 \\
\hline
\end{tabular}

TABLE III

Zeros And Poles of the Transfer Function in ExAmple 2

\begin{tabular}{c|c|c}
\hline$b_{0}$ & zeros & \multicolumn{1}{c}{ poles } \\
\hline 0.015251858 & $1.40060806 \pm 0.52562938 j$ & -0.55252815 \\
& $0.71218856 \pm 1.31038625 j$ & $-0.93489192 \pm 0.14881956 j$ \\
& $-0.34154833 \pm 0.94477549 j$ & $-0.13041701 \pm 0.92361074 j$ \\
& $-0.72080392 \pm 0.77084135 j$ & $0.10577534 \pm 0.70463643 j$ \\
& $-1.07931648 \pm 0.26358750 j$ & $0.38840193 \pm 0.48449893 j$ \\
& $-1.10339797 \pm 0.12036580 j$ & $0.56325419 \pm 0.17664608 j$ \\
\hline
\end{tabular}

comparable. The IIR filter achieves a linear phase response in the passband only approximately. On the other hand, the group delay of the IIR filter can be made lower (11 samples versus 15 samples for the FIR filter). In addition, due to the use of a low-order denominator polynomial $d(z)(r=5)$, the IIR filter can be implemented with comparable number of multiplications but considerably reduced number of additions (and delays) compared with the FIR filter.

Example 2: A stable low-pass IIR filter of order 12 with EPPCLSS was designed to satisfy the following specifications: $\omega_{p}=0.25, \omega_{a}=0.3$, maximum passband deviation $\leq 0.3$ $\mathrm{dB}$, minimum stopband attenuation $\geq 32 \mathrm{~dB}$, group delay in
TABLE IV

PERformance COMPARISONS IN EXAMPLE 2

\begin{tabular}{l|c|c}
\hline Filter & Proposed IIR & Deczky [22] \\
\hline $\begin{array}{l}\text { Maximum passband } \\
\text { amplitude deviation (dB) }\end{array}$ & 0.2709 & 0.4902 \\
\hline $\begin{array}{l}\text { Minimum stopband } \\
\text { amplitude attenuation(dB) }\end{array}$ & 32.1543 & 31.5034 \\
\hline $\begin{array}{l}\text { Maximum group delay } \\
\text { deviation in passband } \\
\text { (Samples) }\end{array}$ & 0.4621 & 0.7130 \\
\hline
\end{tabular}

passband $=9$ samples with the maximum deviation less than 0.5 . With $n=12, r=11, \delta_{p}=0.03, \delta_{a}=0.3, w=30$, and $\varepsilon=8 \times 10^{-4}$, it took the proposed algorithm 27 iterations to converge to a solution point. The poles and zeros of the filter obtained are given in Table III. The largest magnitude of the poles is 0.9467. The performance of the filter is shown in Fig. 2 (solid lines) and Table IV. In the design, the set $\mathcal{S}_{p}$ consists of 38 frequency samples in the normalized passband [0,0.25] and $\mathcal{S}_{a}$ consists of 40 frequency samples in the first third of the normalized stopband $[0.3,0.5]$. The set $\mathcal{S}_{s}$, in this case, consists of 16 equally spaced frequency samples on the entire frequency range $[0,0.5]$ to ensure the filter's stability.

The design obtained was compared to a well-known design by Deczky as [22, Example 1]. The Deczky filter has the same order, $\omega_{p}$ and $\omega_{a}$ as our filter, and its performance is shown in Fig. 2 (dashed lines) and Table IV. It can be observed that our filter has slightly improved performance with a lower and flatter group-delay profile in the passband.

\section{REFERENCES}

[1] A. Antoniou, Digital Filters: Analysis, Design, and Applications, 2nd ed. New York: McGraw-Hill, 1993. 
[2] T. W. Parks and J. H. McClellan, "Chebyshev approximation for nonrecursive digital filters with linear phase," IEEE Trans. Circuits Theory, vol. CT-19, pp. 189-194, Mar. 1972.

[3] T. W. Parks and J. H. McClellan, "A program for the design of linear phase finite impulse response digital filters," IEEE Trans. Audio Electroacoust., vol. AE-20, pp. 195-199, Aug. 1972.

[4] J. F. Kaiser, "Nonrecursive digital filter design using the $I_{0}$-sinh window function," in Proc. IEEE Int. Symp. Circuit Theory, 1974, pp. 20-23.

[5] L. R. Rabiner and B. Gold, Theory and Application of Digital Signal Processing. Englewood Cliffs, NJ: Prentice-Hall, 1975.

[6] A. V. Oppenheim and R. W. Schafer, Discrete-Time Signal Processing. Englewood Cliffs, NJ: Prentice-Hall, 1989.

[7] D. C. Farden and L. L. Scharf, "Statistical design of nonrecursive digital filters," IEEE Trans. Acoust., Speech, Signal Processing, vol. ASSP-22, pp. 188-196, June 1974.

[8] V. R. Algazi and M. Suk, "On the frequency weighted least-squares design of finite duration filter," IEEE Trans. Circuits Syst., vol. CAS-22, pp. 943-953, Dec. 1975.

[9] V. R. Algazi, M. Suk, and C. S. Rim, "Design of almost minimax FIR filters in one and two dimensional by WLS technique," IEEE Trans. Circuits Syst., vol. CAS-33, pp. 590-596, June 1986.

[10] Y. C. Lim, J. H. Lee, C. K. Chen, and R. H. Yang, "A weighted leastsquares algorithm for quasiequiripple FIR and IIR digital filter design," IEEE Trans. Acoust, Speech, Signal Processing, vol. 40, pp. 551-558, Mar. 1992.

[11] S. C. Pei and J. J. Shyu, "Design of arbitrary FIR log filters by weighted least-squares technique," IEEE Trans. Signal Processing, vol. 42, pp. 2495-2499, Sept. 1994.

[12] J. W. Adams, "FIR digital filters with least-squares stopbands subject to peak-gain constraints," IEEE Trans. Circuits Syst., vol. 38, pp. 376-388, Apr. 1991.

[13] J. W. Adams, J. L. Sullivan, R. Hashemi, C. Ghadimi, J. Franklin, and B. Tucker, "New approaches to constrained optimization of digital filters," in Proc. IEEE Int. Symp. Circuits and Systems, 1993, pp. 80-83.

[14] J. W. Adams, P. Kruethong, R. Hashimi, J. L. Sullivan, and D. Gleeson, "New quadratic programming algorithms for designing FIR digital filters," in Proc. 27th Asilomar Conf., 1993, pp. 1206-1210.

[15] J. L. Sullivan and J. W. Adams, "A new nonlinear optimization algorithm for asymmetric FIR digital filters," in Proc. IEEE Int. Symp. Circuits and Systems, May 1994, pp. 541-544.

[16] W.-S. Lu, S.-C. Pei, and C.-C. Tseng, "A weighted least-squares method for the design of stable 1-D and 2-D IIR digital filters," IEEE Trans.
Signal Processing, vol. 46, pp. 1-10, Jan. 1998.

[17] W.-S. Lu, "Design of stable IIR digital fitters with equiripple passbands and peak-constrained least-squares stopbands," in Proc. ISCAS'97, pp. 2192-2195.

[18] M. C. Lang, "Weighted least squares IIR filter design with arbitrary magnitude and phase responses and specified stability margin," in Proc. 1998 IEEE Symp. Advances in Digital Filtering and Signal Processing, Victoria, BC, pp. 82-86.

[19] P. A. Regalia, Adaptive IIR Filtering in Signal Processing and Control. New York: Marcel Dekker, 1995.

[20] A. T. Chottera and G. A. Jullien, "A linear programming approach to recursive digital filter design with linear phase," IEEE Trans. Circuits Syst., vol. CAS-29, pp. 139-149, Mar. 1982.

[21] R. Fletcher, Practical Methods of Optimization, 2nd ed. New York: Wiley, 1987.

[22] A. G. Deczky, "Synthesis of recursive digital filters using the minimum p-error criterion," IEEE Trans. Audio Electroacoust., vol. AE-20, pp. 257-263, 1972

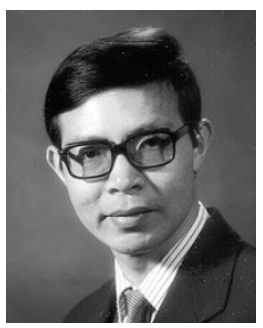

W.-S. Lu (S'81-M'85-SM'90-F'99) received the B.S. degree in mathematics from Fudan University, China, the M.S. degree in electrical engineering and the Ph.D. degree in control science, both from the University of Minnesota, Minneapolis, in 1964 1983, and 1984, respectively.

He was a post-doctoral Fellow at the University of Victoria, Victoria, BC, Canada, in 1985 and a Visiting Assistant Professor at the University of Minnesota in 1986. Since 1987, he has been with the University of Victoria, where he is currently a Professor. His teaching and research interests are in the areas of digital signal processing and numerical optimization. He is the co-author, with A. Antoniou, of Two-Dimensional Digital Filters (New York: Marcel Dekker, 1992).

Dr. Lu served as an Associate Editor of the Canadian Journal of Electrical and Computer Engineering in 1989, and then as Editor from 1990 to 1992 . He was an Associate Editor of IEEE TRANSACTIONS ON CiRCUITS AND Systems II from 1993 to 1995. Presently, he is an Associate Editor for both the IEEE TRANSACTIONS ON CIRCUITS AND SySTEMS I and the International Journal of Multidimensional Systems and Signal Processing. 